logischen Kenntnissen vereint; mit dem Schiffe, welches ein einziges Mal jährlich die Ostküste Grönlands befährt, hat er beständig teils Aufzeichnungen über Vögel, teils Vogelbälge an mich gesandt; was er mir auf diese Weise mitgeteilt, habe ich zweimal veröffentlicht und hoffe, im Laufe des Winter einen Bericht auszuarbeiten über das, was ich in den letzten drei Jahren erhalten habe.

Hiermit werde ich mir erlauben $\mathrm{zu}$ schliessen; was ich gesagt habe, ist sehr unvollständig; ich hoffe indess, das Wesentlichste vorgeführt zu haben, so dass mein Vortrag eine Vorstellung vom Vogelleben in Grönland geben konnte.

\title{
Beiträge zur Akustik des Stimmorgans der Sperlingsvo̊gel.
} Von Karl Deditius.

Seit Jahrhunderten wenden die Forscher dem Stimmorgan der Vögel ihre Aufmerksamkeit $\mathrm{zu}$, doch mehr in anatomischer. als in akustischer Hinsicht. Das Ergebnis der akustischen Forschungen dürfte wohl deshalb hinter den Erwartungen zurückgeblieben sein, weil zu den Versuchen gewöhnlich nur entnommene Stimmorgane verwendet wurden. Da solche ihre ursprüngliche Beschaffenheit schon mit dem Tode des Vogels verlieren, so werden sie zu akustischen Versuchszwecken ungeeignet. Die Akustik des Stimmorgans kann indes auf andere Weise ermittelt werden, denn Tonwerkzeuge, die ihm in der Anordnung entsprechen, geben schon reichlichen Einblick in die Geheimnisse der Stimme. $\mathrm{Zu}$ Versuchszwecken erwies sich als am meisten geeignet das Blechblasinstrument, weil es in akustischer Hinsicht dem Stimmorgan der Vögel am nächsten steht. Die wenigen Beiträge, die ich durch Vergleiché mit dem Blechblasinstrument gewonnen habe, sollen auf weitere Ermittelungen anregend wirken, zumal auf dem Gebiete noch Vieles nachzuholen ist.

Die Stimmorgane der vielen stimmbegabten Vögel sind nicht gleich, und ich beschränke mich deshalb nur auf die Sperlingsvögel, deren Stimmorgane ich von 50 Arten gesammelt habe. Nur hin und wieder war 'es zweckmässig, anch andere Vögel in Vergleich zu ziehen.

Zunächst wird die akustische Verwandtschaft des Blasinstruments mit dem Stimmorgan des Vogels nachzuweisen sein. Das Blasinstrument besteht aus dem an einem Ende erweiterten 
Schallrohr und aus dem Mundstück. Durch Einstecken in das enge Ende des Rohres wird die Verbindung zu einem Stück hergestellt. Der obere Teil des Mundstücks gleicht einem Kessel mit durchlochtem Boden, an welchen sich ein kegelförmig ausgebohrter Zapfen anschliesst. Zur Tonerzeugung sind noch eine Windlade mit Windrohr und schwingungsfähige Zungen nötig, die der Bläser mit den Lungen, der Mundhöhle und den Lippen ersetzt. Das Stimmorgan des Vogels dagegen besitzt alle zur Tonbildung erforderlichen Teile. Die Lungen mit den Luftsäcken dienen als Windladen, die Bronchien als Windröhren, die Labien und die innere Paukenhaut der Bronchien als schwingende Zungen, die Trommel als Mundstück, die Luftröhre als Schallrohr, der mit der Trommel verbundene engste Teil der Luftröhre ist zu vergleichen mit dem Zapfen des Mundstücks und der obere Kehlkopf sowie die Mundhöhle mit dem erweiterten Ende des Schallrohrs.

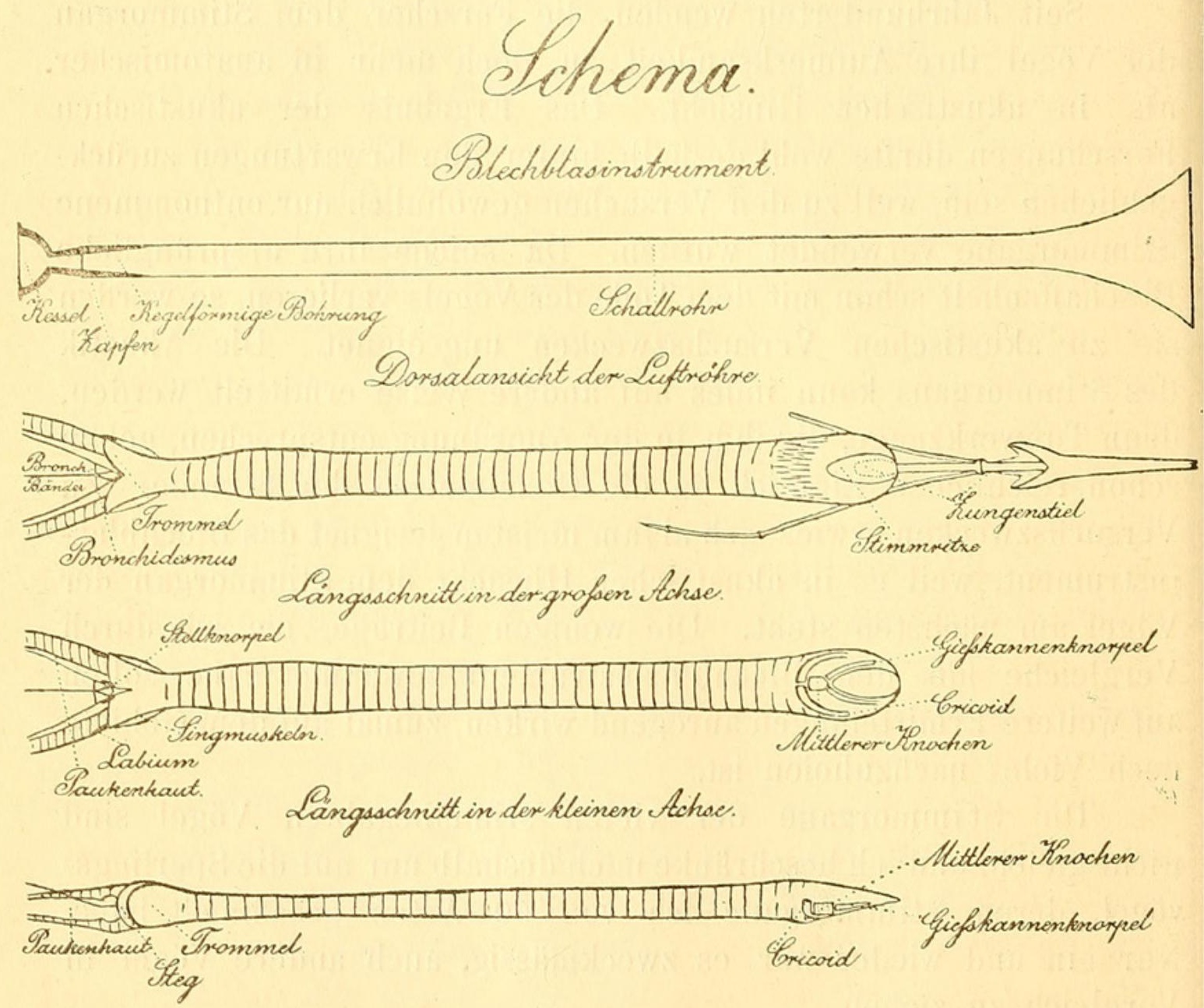

Im Blasinstrument geht die Tonbildung in folgender Weise vor sich. Der Bläser drückt zunächst die fest auf einander gepressten Lippen an das Mundstück. Ein aus den Lungen 
getriebener Luftstrom wird vor den geschlossenen Lippen aufgehalten und in der Mundhöhle so lange durch nachströmende Luft verdichtet, bis er die nötige Stärke erreicht hat, um zwischen den Lippen durchzubrechen. Es entsteht ein Spalt, aus dessen Rändern sich weiche, in den Kessel ausschlagende Zungen bilden. Die einseitige Bewegung kann nur einen Augenblick anhalten, denn schon in dem nächsten werden die Lippen durch eigene Elastizität in die frühere Lage gebracht, womit sie den Spalt schliessen. Die nachströmende Luft öffnet den Spalt wieder, und so wiederholt sich dieses abwechselnde Spiel in schneller Folge viele Mal hintereinander. Zur unmittelbaren Tonerzeugung sind die weichen Lippenränder jedoch nicht fähig, weil sie der geringen Elastizität wegen weder schnell genug noch für die Dauer regelmässig schwingen können. Bei zweckmässigem Anblasen entstehen jedoch wechselnde Schwingungsverhältnisse der Lippen, in welchen auch regelmässige Schwingungen vorkommen. In solchem Falle nimmt die in der Röhre eingeschlossene Luftröhre die letzteren Schwingungen an und schwingt in regelmässigen, pendelartigen Bewegungen mit. Diese Bewegungen bleiben bestehen, solange der Luftstrom anhält, wobei die mächtigen Schallwellen die Lippen $z u$ gleich schnellen Bewegungen zwingen. Aus den wechselseitigen Bewegungen entsteht ein Ton, den aber nicht die zu Zungen gestalteten Lippen erzeugen, sondern die Luftsäule.

Im Stimmorgan des Vogels entsteht der Ton in gleicher Weise. Es ist bekannt, dass die Labien mittels eines der oberen Bronchialhalbringe quer in das Lumen gezogen und dass die innere Paukenhaut der Bronchien durch Hebung der dachförmig herabhängenden Stellknorpel dem vorgezogenen Labium so genähert werden können, dass beide Teile aneinander stossen. In diese Stellung werden sie durch Spannung mittels der Brustbeinmuskeln und der sogenannten Singmuskeln gebracht. Wahrscheinlich beteiligen sich dabei auch Bänder, die mitten vom Bronchidesmus ausgehen und die einerseits mit den Stellknorpeln und anderseits mit den Luftsäcken in Verbindung stehen. Das Gesangsvermögen der Vögel pflegt man nach der Entwickelung der Singmuskeln zu schätzen; doch ist diese Voraussetzung irrig. Zwar besitzen viele gute Sängerarten stark entwickelte Muskeln an der Trommel (Singmuskeln), aber ebenso auch viele schlechte Sängerarten und sogar Vögel, die ausser dem Lockruf andere Töne nicht von sich geben können. Wiederum giebt es gute und schlechte Sänger 
mit nur schwach entwickelten Singmuskeln. Demnach dürfte diese Bezeichnung nicht zu Recht bestehen, der Name „Trommelmuskeln" wäre zutreffender. Wie der Bläser die Lippen fest aufeinander pressen muss, so wird auch der Vogel die Stimmbänder ganz dicht aneinander stossen müssen; denn geschähe dies nicht, so könnten die abwechselnd $\mathrm{zu}$ öffnenden und $\mathrm{zu}$ schliessenden Spalte und die daraus folgenden Luftstösse nicht entstehen. Sobald nun ein Luftstrom gegen die geschlossenen Stimmbänder getrieben wird, hält er dort so lange an, bis ihm die nachströmende Luft eine solche Dichtigkeit giebt, dass er durchbrechen kann; der erforderliche Zeitraum ist nur sehr kurz. Ist der Durchbruch erfolgt, dann wird der Spalt durch die Elastizität der Stimmbänder sofort geschlossen, durch nachströmende Luft aber auch sofort wieder geöffnet. Das abwechselnde Entstehen und Schliessen des Spalts wiederholt sich hintereinander schnell viele Mal, und daraus entsteht eine Reihe von Luftstössen, deren Summe einen Ton giebt. Auch von den Stimmbändern der Vögel ist nicht anzunehmen, dass sie regelmässig und schnell genug schwingen, um selbstständig Töne, wie harte Metallzungen, erzeugen zu können, denn dafür sind sie zu weich. Immerhin dürften ihre Bewegungen schneller und regelmässiger ausfallen als bei den weniger elastischen menschlichen Lippen. Deshalb werden passende Schwingungen, die sich mit denjenigen in der Luftröhre vereinigen können, in reichlichem Masse vorhanden sein. Dadurch wird die Ansprache der Luftsäule leichter und die aufzuwendende Kraft geringer sein. Auch hier sind es nicht die schwingenden Stimmbänder, die den Ton erzeugen, sondern nur die in der Luftröhre eingeschlossene Luftsäule ist es. Da nur die Luftsäule tönt, so bleibt es gleich, ob der Vogel, wie alle Sperlingsvögel, zwei Paar Stimmbänder besitzt oder nur ein Paar, wie die Papageien, denn sie zwingt ja ihre regelmässigen Schwingungen den unregelmässigen der Stimmbänder auf. Ferner bleibt es gleich, ob die Luftröhre grade, gebogen oder in Windungen liegt, weil durch die Form das Volumen der Luftsäule nicht geändert wird. Unmittelbaren Beweis liefert das Blasinstrument, welches nur der bequemen Handhabung wegen gewunden wird. Die Windungen können ganz beliebig sein.

Fast alle schwingenden Körper, in unserem Falle die in der Luftröhre eingeschlossene Luftsäule, geben nicht einfache Schwingungen, sondern es treten mit den langsameren gleich- 
zeitig die in dem Körper überhaupt möglichen Schwingungen auf, deren Dauer die Hälfte, ein Drittel, ein Viertel u. s. w. ist. Den tiefsten, aus den langsamsten Schwingungen entstehenden Ton nennt man den Grundton, die ihn begleitenden höheren die Obertöne. Je länger die Luftsäule ist, in um so mehr schwingende Teile kann sie sich zerlegen und demgemäss auch viele Obertöne bringen, da jede Teilschwingung ihren Oberton erzeugt. Mit A bnahme der Länge nimmt auch die Anzahl der Teilschwingungen $a b$, und mit ihnen die der Obertöne. Schliesslich hören die Teilschwingungen und Obertöne auf. Röhren in Länge der Luftröhre kleinerer Vögel sind für Teilschwingungen schon viel $\mathrm{zu}$ kurz, sobald die Wand hart ist. Aus den weichen Luftröhren kleinerer Singvögel hören wir jedoch die Tonintervalle der Terzen, der Quarte, Quinte, und sogar das der Octave im Lockruf der Nachtigall. Der Vogel vermag zwar die Luftröhre zu verlängern und damit die Töne zu erniedrigen, doch nicht in dem für die erwähnten Intervalle benötigten Masse. Zur Erniedrigung des Tones um eine Octave müsste die Nachtigall ihre Luftröhre in der Länge verdoppeln, und das ist doch unmöglich. Nach meiner Ansicht wird das Auftreten von Obertönen in den Luftröhren kleinerer Vögel von der Schlaffheit der Wand begünstigt. Die innere Schleimhaut und die Bindehäute zwischen den Knochenringen setzen den Schallwellen nur geringen Widerstand entgegen Ihre Bewegungen werden langsamer als in harten Röhren, und demgemäss fallen die Töne tiefer aus. In Betreff der Tonhöhe kann man die Luftröhre einer erheblich längeren harten Röhre gleichstellen, deren Länge für Teilschwingungen noch ausreichend ist. So erkläre ich mir das Auftreten von Obertönen in den kurzen Luftröhren der kleineren Vögel.

Für die Anzahl und Höhe der Obertöne in Röhren ist deren Länge und Weite massgebend. Auf sehr engen Röhren sprechen der Grundton und die tieferen Obertöne nicht an; auf Röhren, die an dem einen Ende eng, an dem anderen weit sind, spricht der Grundton nicht immer an und die hohen Obertöne sind nicht so zahlreich wie auf der engen Röhre; auf weiten Röhren sprechen der Grundton und tiefere Obertöne an, die hohen fallen aus. Der Klang einer engen Röhre ist demnach leer, doch scharf, der einer weiten Röhre dagegen dumpf; am vollsten und auch am angenehmsten ist er auf der allmählich sich erweiternden Röhre. Alle diese Formen sind in den Luft- 
röhren der Vögel zu finden. Das Auftreten jener Obertöne ist aber von Bedingungen abhängig. Nicht nur die Stärke des Anblasens, sondern auch die anfängliche Weite des Ansatzrohres sind dafür entscheidend. Die Versuche auf dem Blechinstrument ergeben, dass vorzugsweise die hohen Obertöne auftreten, wenn der kegelförmig ausgebohrte Zapfen lang ist und mit einer kleinen Öffnung nach dem Mundstückkessel endigt und dass die hohen Obertöne wegfallen, wenn der Zapfen kurz und die Öffnung zum Kessel weit ist. Die gleichen Wahrnehmungen sind bei den Luftröhren der Vögel zu machen. Diejenigen der Feldlerche, des Rotkehlchens, des Buchfinken sind anfänglich bedeutend enger als in der Folge, ihre Stimmen sind daher reicher an Obertönen als diejenigen der Grasmücken, der Haidelerche, des Baumpiepers, bei welchen Vögeln der Anfang der Luftröhre nur wenig enger ist als die Fortsetzung. Die Stimme der ersteren Vögel ist hart, der höheren Obertöne wegen, die der letzteren weich.

Die Stimme einer jeden Vogelart besitzt ihre besondere Klangfarbe. Dies ist jene Eigentümlichkeit, durch welche Töne von gleicher Höhe unterschieden werden. Selbst ein ungeübtes Ohr empfindet, ob der Ton z. B. von einer Violine, einer Trompete, einer Flöte $u$. s. w. ausgegangen ist. Die Verschiedenheit solcher Empfindung beruht hauptsächlich auf dem Mitklingen von Obertönen, zum Teil auch auf der Beimischung von schwachen Geräuschen. In regelmässigen Röhren treten die Obertöne gleichmässig auf, in unregelmässigen können sie es nicht, weil die Schwingungsformen Änderungen erleiden, welche die Obertöne beeinflussen. Gewisse Obertöne werden übermässig verstärkt, gewisse geschwächt und gewisse sogar unterdrückt; der Wechsel in der Klangfarbe kann durch die unbeschränkte Anzahl von Schwingungsformen sehr mannigfaltig sein. Regelmässige Luftröhren, das wären cylindrische oder solche, deren Wand sich in der Kurve einer Parabel erweitert, finden wir bei keinem Vogel. Die meisten Sänger besitzen eine anfänglich im Querschnitt runde Luftröhre, die bald auf eine kurze Strecke hin platt wird und im übrigen Teile elliptischen Querschnitt annimmt, der sich vor dem oberen Kehlkopfe erweitert. Die Abplattung, die nicht alle Vögel aufweisen, und die Achsen der Ellipsen wechseln bei allen Vogelarten, die Obertöne treten demnach verschieden auf und aus ihnen entsteht die verschiedene Klangfarbe. 
Die Röhre des Blasinstruments sucht man je nach der Art des Instruments möglichst gleichmässig zu gestalten, damit die Obertöne auch gleichmässig bleiben; die Klangfarbe wird indes durch das Mundstück geändert. Auf derselben Röhre werden Mundstücke mit gleicher Bohrung des Zapfens, jedoch mit verschiedenen Kesselformen, wohl die gleichen Obertöne ansprechen lassen, ihre Klangfarbe wird aber durch jedes dieser Mundstücke eine andere sein. Im Allgemeinen geben flache Kessel harte und tiefe Kessel weiche Klangfarben, wobei die Kesselweite die Tonstärke bestimmt. Es lassen sich eine erhebliche Anzahl Zwischenformen mit immer anderer Klangfarbe schaffen, da selbst ganz geringe Abweichungen die Klangfarbe beeinflussen. Im Stimmorgan des Vogels nimmt die Trommel die Rolle eines Mundstücks ein, sie trägt also auch zur Änderung der Klangfarben bei. So wie die Luftröhre der einen Vogelart nie genau derjenigen einer anderen Art gleicht, so wechseln auch die Trommeln der vielen Arten, und jede Trommel ändert die Klangfarbe in eigener Weise. Wird nun in Erwägung gezogen, dass jede Luftröhre ihre besonderen Obertöne besitzt, dass deren Erweckung von der anfänglichen Weite abhängt, dass die Trommel selbständig die Klangfarbe ändert und dass endlich die Anzahl der auftretenden Obertöne auch von der Stärke des Anblasens bestimmt wird, so muss die Klangfarbe in den Stimmorganen der Vögel eine sehr mannigfaltige sein, und das ist in der That der Fall.

Was der Änderung der Klangfarbe durch Mundstücke zu Grunde liegt, darüber verlautet nirgends etwas. Es ist wahrscheinlich, dass die in dem Mundstücke - beim Vogel in der Trommel - eingeschlossenen, weiten Schallwellenteile nicht in vollem Umfange von dem anfänglich sehr engen Zapfen aufgenommen werden, so dass im Kessel ein Überschuss verbleibt, der Schallreflexe erzeugt, die sich den Tönen in der Röhre beimischen und damit die Klangfarbe beeinflussen. Der Einfluss wird um so bemerklicher, je weiter der Kessel ist. ${ }^{1}$ ) Es ist zweifelhaft, ob die sehr kurzen Luftröhren der kleinsten Vögel noch fähig sind, Obertöne zu bilden. Wenn der Zweifel berechtigt wäre, dann könnte es nur die Trommel sein, die solchen Luftröhren die Klangfarbe giebt.

1) Auch die morgagnischen Taschen im Kehlkopfe des Menschen dürften nicht ohne Einfluss auf die Klangfarbe der Stimme sein, da in jenen ebenfalls Schallreflexe vorkommen können. 
Statt der Trommel besitzen die Enten zwei seitliche, nicht gleich grosse und unregelmässige Ausbuchtungen. Die Schwingungen der Stimmbänder teilen sich diesen Hohlräumen mit, worin dann unregelmässige Schwingungen entstehen, die nicht Töne, sondern Geräusche bilden. Letztere fliessen in die Luftröhre ab, welche sie durch Resonanz verstärkt. Daher kann die Stimme der Enten sich nur in heiseren Geräuschen und nicht in Tönen äussern.

Die Vögel bringen nicht nur Töne sondern auch Geräusche d. s. Gekreische, Warnungsrufe und unmelodische Beigaben im Gesange. Ein Geräusch entsteht aus unregelmässigen Schwingungen, während der Ton nur aus regelmässigen Schwingungen des tönenden Körpers entstehen kann. Die gleichmässig in der Luftröhre schwingende Luftsäule erzeugt nur Töne. Wenn ausser solchen noch Geräusche entstehen, so können diese nur durch Störungen der Schallwellen gebildet werden. Das Hervorbringen der Geräusche schreibt man gewöhnlich der Zunge $\mathrm{zu}$, doch $\mathrm{zu}$ Unrecht. Sie ist bei den Sperlingsvögeln nicht nur steif, sondern sie liegt im offenen Raume auch zu weit entfernt von der Stimmritze, um störenden Einfluss ausüben zu können. Deshalb tritt der Ton zum grossen Teile schon in die freie Luft, bevor er die Zunge erreicht. Durch ihre Vorlagerung wird nur die Schallausbreitung ein wenig gehindert, der Ton verliert an Stärke und kann in Folge Beugung des Zungenstiels durch Schallreflexe etwas in der Klangfarbe leiden. Die platte und dünne Zunge der Insektenfresser hindert die Schallausbreitung weniger als die dickere Zunge der Körnerfresser, darum ist die Stimme der ersteren in der Regel klangvoller. Eine Ausnahme in beschränktem Masse macht der Graupapagei mit seiner weichen und allseitig beweglichen Zunge. Ein zuverlässiger Beobachter teilte mir mit, dass sein Graupapagei während der Nachahmung menschlicher Laute nur dann sich der Zunge bedient, wenn er das $r$ sprechen will. Er stösst in solchem Falle die Zunge ebenso an den Schnabel wie sie der Mensch bei diesem Laute an den Gaumen stossen muss. Die sprechenden Sperlingsvögel, wie Stare, Elstern und Nussheher, besitzen nur steife Zungen, die Nachahmung der Sprachlaute fällt ihnen daher schwerer. Da der Ton weder mit der Zunge noch in der Mundhöhle zum Geräusch umgewandelt werden kann, so muss letzteres schon im oberen Kehlkopfe entstehen. Dieser erfüllt auch die Bedingungen hierzu. Wir bemerken in 
ihm eine eigentümliche, mit einer Schleimhaut überzogene Knochengruppe, die bisher wenig Beachtung gefunden hat. An den Seiten der Stimmritze liegen die beiden Giesskannenknorpel; mit ihren Enden stossen sie, durch Gelenkbänder verbunden, an einen hohen, sehr dünnwandigen Knochen in Schildform (Cricoid), und hinter diesem liegen noch zwei platte Knochen in Rippenform. Diesem letzteren Paar lege ich grosse Bedeutung für die Stimme bei. Es ist anfänglich mit dem Kehlkopfe verwachsen, die Fortsetzung bis etwa zur Mitte ruht lose auf der Kehlkopfwand, und der Schluss hebt sich im Bogen aufwärts strebend davon ab, bis die Enden hinter dem Cricoid zusammenstossen. Unter dem Cricoid liegen zwei Muskelbündel, die ihre Fasern nach verschiedenen Richtungen abgeben; die meisten entfallen auf das Cricoid selbst. Die Menge der Muskelfasern lässt auf grosse Beweglichkeit dieses mittleren Knochen schliessen. Der Vogel wird damit die an den Knochenstücken haftende, dazwischen aber schlaft herabhängende Schleimhaut durch Hebung und Senkung, vielleicht auch durch seitliche Verschiebung, in mannigfacher Weise spannen und den Ausdruck des hier entstehenden Geräusches beliebig einrichten köunen. Ja nach der Art des Vogels wechselt die Gestalt dieser Knochen, besonders die des mittleren. Meist tritt er in Schildform auf, manchmal auch als Ring oder als Dreieck. Auch hier macht sich der Widerstand geltend, den die schlaff hängende Haut der Wellenbewegung entgegengesetzt, denn sie ist wesentlich schlaffer als im übrigen Teile des oberen Kehlkopfes und in der Luftröhre und demnach ist der Widerstand auch viel geringer. Die Schallwellen können sich an dieser Stelle nicht so schnell wie in der Luftröhe bewegen, und unregelmässige Bewegungen lassen nicht Töne, sondern nur Geräusche entstehen. Um Töne hervorzubringen, muss der Vogel die rippenförmig gebogenen Knochen einwärts wenden. Damit wird die schlaffe Haut gespannt und mit den gebogenen Knochenstücken an die Kehlkopfwand gelegt. Die Bewegungsfähigkeit dieses Knochenpaares ist nicht anzuzweifeln. Vor ihrer Wendung bieten sie den Schallwellen noch einiges Hindernis, danach nicht mehr.

Jene sonderbare Einrichtung im oberen Kehlkopfe benutzen begabte Vögel zur Nachahmung menschlicher Sprachlaute. Die Fertigkeit, die der Mensch in der Zunge und in den Zähnen besitzt, um die von den Stimmbändern gebildeten Töne in Sprach- 
laute umzuwandeln, die grösstenteils auch Geräusche sind, besitzen einige Vogelarten im oberen Kehlkopfe.

Die Stärke eines Tones hängt zunächst von dem Kraftaufwande $a b$, mit dem er erzeugt wurde, und dann von der Weite der Schallwellen, (die Länge ist nur für die Tonhöhe massgebend). Im Stimmorgan schwingen die Stimmbänder und mit ihnen die Luftsäule um so heftiger, je stärker der Luftstrom ist. Die Schwingungszahl und die ihr entsprechende Tonhöhe bleiben dabei unverändert. Bedingung ist hierbei ein ungestörter Abfluss der Schallwellen in die freie Luft. Die Stimme eines Vogels wird nie laut sein, doch die gleiche Tonhöhe behalten, sobald er die Stimmritze verengt oder gar den Schnabel schliesst. Sofern der Schallabfluss nicht beeinträchtigt wird, geben die weiten Röhren starke und die engen Röhren schwache Töne. Anfänglich enge und dann allmäblich sich erweiternde Röhren, auch solche, von denen nur ein Ende erweitert ist, tönen ziemlich stark. Hingegen tönen nur schwach diejenigen Röhren, welche an beiden Enden eng, doch in der Mitte weit sind. Die Tonstärke enger Röhren kann leicht überschätzt werden. Ihr Grundton und die tieferen Obertöne sprechen entweder nur schwach oder gar nicht an, wogegen die hohen Obertöne stärker hervortreten. Da Schallwellen hoher Obertöne nur klein sind, so dringt verhältnismässig viel davon in die Ohröffnung, während von den weiten, stärkeren Schallwellen ein nur geringer Teil vom Ohr aufgenommen werden kann. Höhere Töne werden daher für das Ohr empfindlicher als tiefere. Aus diesem Grunde kann die schwache Stimme kleinerer Vögel dem Ohr eindrucksvoller sein als die starke Stimme grösserer Vögel. Der grösseren Tonstärke wegen hört man auch die Stimme der letzteren auf weitere Entfernung als die der ersteren. Die Vögel sind im Stande, die Stärke der Stimmen abzustufen. Wie sie diese schwächen, bemerkte ich vorhin. Die Verstärkung bewirken sie durch kräftigeren Antrieb der Stimmbänder und auch durch Erweiterung des oberen Kehlkopfes. Die Erweiterung tritt ein durch Wendung der rippenförmigen Knochen und durch Beugung des unteren Teiles vom Zungenstiel, dessen Ende mit dem Kehlkopfe verwachsen ist. Die Erweiterungen machen sich beim singenden Vogel schon äusserlich bemerkbar. Man nimmt gewöhnlich an, dass dies Aufblähungen wären. Solche können es aber nicht sein, denn bei geöffneter Stimmritze ist eine Ansammlung und Verdichtung der Luft im Kehlkopfe selbstver- 
ständlich unmöglich. Eine weitere und wohl die erheblichste Tonverstärkung findet in der Mundhöhle durch Schallreflexe statt, wobei mehr die Tiefe als die Weite massgebend ist. Die Mundhöhle der Insektenfresser ist tiefer als diejenige der Körnerfresser, daher der ersteren Stimme auch stärker.

Die Höhe der Töne kleinerer Vögel nach dem Gehör zu bestimmen, dürfte kaum gelingen, weil das Ohr kleine Intervalle in hohen Tonlagen nicht mehr zu unterscheiden vermag, es irrt sogar im grössten Tonintervall, der Oktave. Die Höhe langer, anhaltender Töne liesse sich vielleicht mittels Syrene ermitteln. Abhängig ist die Tonhöhe von der Schwingungszahl der in der Luftröhre eingeschlossenen Luftsäule; je grösser die Anzahl ist, desto höher wird der Ton. Würde der Vogel die Luftröhre nicht verlängern, so könnte er nur den Grundton und wenige Obertöne hervorbringen. Es giebt auch Vögel, die kaum über denselben Ton hinauskommen; wahrscheinlich sind sie nicht fähig, die Luftsäule zu verlängern. Dagegen dürften die Sänger die Luftröhre bis zu einer bestimmten Grenze nach Belieben verlängern, und auf die Verlängerung muss auch die Verkürzung folgen. In diesem Wechsel erhält die Luftröhre zahlreich verschiedene Längen, von welchen jede ihren besonderen Grundton und die davon abhängigen Obertöne führt. Demnach ist die Luftröhre in einem begrenzten Tonumfange an Tonintervallen sehr reich. Gewöhnlich begnügt sich der Vogel mit den von seiner Art angenommenen Weisen, doch gehen manche Vögel darüber hinaus, indem sie in ihre Weisen auch solche anderer Arten einflechten, ohne gerade Spötter zu sein. Aufgezogene Nestjunge nehmen leicht allerlei Töne an, die sonst ihrer Art ganz fremd sind, was beweist, dass die Vögel zur Hervorbringung sehr vieler Töne fähig sind. Ausgiebigen Gebrauch von ihren Fähigkeiten machen schon in der Freiheit die Spötter. Ich besass Wildlinge von rotrückigen Würgern, die zehn Gesänge anderer Vögel vollständig wiederzugeben vermochten und ausserdem ebensoviel Bruchstücke von Gesängen und Rufen wieder anderer Vögel. In allen diesen Nachahmungen fehlte aber selbstverständlich die Klangfarbe der betreffenden Arten. Die Luftröhre der Würger zeigt eine regelmässigere Form als die der anderen Singvögel. Sie erweitert sich von der Trommel ab bis zum oberen Kehlkopfe hin allmählich und ist auch nirgends abgeplattet, demnach kann sie für die Bildung vieler Töne mehr 
geeignet sein. Die Wiedergabe von Tönen aus längerer Luftröhre ist leicht erklärlich, da hierzu der Vogel seine kürzere Luftröhre nur entsprechend auszuziehen hat. Dagegen ist die Wiedergabe von Tönen kürzerer Luftröhren eine bewundernswerte Kunstleistung. Um die höheren Töne zu treffen, muss der grössere Vogel den Grundton seiner Luftröhre durch Verlängerung so einstellen, dass die Obertöne davon mit der Höhe des Tones der kurzen Luftröhre übereinstimmen. So oft die letztere den Ton wechselt, so oft muss auch der Grundton der längeren Luftröhre gewechselt werden. Und das Alles vollzieht sich mit so grosser Schnelligkeit und Sicherheit, dass man über die musikalische Begabung der Vögel staunen muss.

Auch in der Umstimmung der Luftröhre nähert sich das Stimmorgan sehr dem Blasinstrument, dessen Grundton durch Einschaltung von Ventilzügen mehrfach erniedrigt wird. Aus den Obertönen der erniedrigten Grundtöne werden die Tonleitern zusammengestellt. Bei der Posaune gestaltet sich die Einstimmung der Grundtöne und ihrer Obertöne noch einfacher durch Auszug der Röhre. Diese Art Blechinstrument steht also dem Stimmorgan am nächsten.

Alle Versuche, den Naturgesang der Vögel in musikalische Notenschrift zu setzen, waren bisher vergeblich, und sie werden es wohl auch bleiben, weil die Vögel Tonintervalle unserer Musik nicht bringen. Das kleinste musikalische Tonintervall ist der Halbton; wie klein sind dagegen die Tonabstufungen, die aus den verschiedenen Verlä ngerungen und rückwärtigen Verkürzungen der Luftröhre folgen! Nun richtet sich die Länge und Tontiefe der Luftröhre etwa nach der Grösse des Vogels. Die Grundtöne, Obertöne und Abstufungen durch Verlängerung können daher bei den vielen Vogelarten nicht gleich sein. Durch Abrichtung sind indess Vögel auch an musikalische Tonintervalle zu gewöhnen. In begrenztem Tonumfange sind die stimmbegabten Vögel zur Wiedergabe solcher Intervalie wohl befähigt.

Die Anzahl der Schwingungen folgt im Allgemeinen aus dem Spannungsgrade der Stimmbänder. Bei schwacher Spannung treibt sie der Luftstrom weiter auseinander als bei starker. Der Spalt wird gross, die schwingenden Teile sind schwer und bewegen sich nur langsam. Ist aber die Spannung stark, dann entsteht ein kleinerer Spalt, es können nur kleinere Teile schwingen, und da diese leichter sind als die grossen, so fallen ihre Bewegungen 
schneller aus. Der Anzahl der Schwingungen entsprechend wird im ersteren Falle der Ton tief, im letzteren hoch sein. Indess vermag der Vogel die Tonhöhe nicht beliebig zu bestimmen, sofern er nicht die Länge der Luftrohre ändert, denn aus der Länge folgt die Höhe des Grundtones und die der Obertöne, dazwischen liegende Tonintervalle sprechen nicht an. Daher ist eine genau bemessene Spannung der Stimmbänder auch nicht erforderlich. Für den Grundton und für jeden Oberton reichen schon annähernd richtige Spannungen aus. Die geringen Tonänderungen, die aus Verlängerungen und Verkürzungen der Luftröhre folgen, werden in den meisten Fällen keiner besonders zu bemessenden Spannung der Stimmbänder bedürfen, weil die Luftsäule in solchen Fällen die Schnelligkeit der Schwingungen sofort ändert. Auch über diese Punkte giebt uns das Blasinstrument Aufschluss. 


\section{$2 \mathrm{BHL}$ Biodiversity Heritage Library}

1902. "Beiträge zur Akustik des Stimmorgans der Sperlingsvögel." Journal fu

r Ornithologie 50, 101-113. https://doi.org/10.1007/bf02286799.

View This Item Online: https://www.biodiversitylibrary.org/item/104733

DOI: https://doi.org/10.1007/bf02286799

Permalink: https://www.biodiversitylibrary.org/partpdf/142580

\section{Holding Institution}

Smithsonian Libraries

\section{Sponsored by}

Biodiversity Heritage Library

\section{Copyright \& Reuse}

Copyright Status: Public domain. The BHL considers that this work is no longer under copyright protection.

This document was created from content at the Biodiversity Heritage Library, the world's largest open access digital library for biodiversity literature and archives. Visit BHL at https://www.biodiversitylibrary.org. 